\title{
Robust Age at Onset Linkage Analysis in Nuclear Families
}

\author{
A. Callegaro J.C. van Houwelingen J.J. Houwing-Duistermaat \\ Department of Medical Statistics and Bioinformatics, Leiden University Medical Center, University of Leiden, \\ Leiden, The Netherlands
}

\section{Key Words}

Additive gamma frailty model $\cdot$ Log-normal frailty model . Retrospective likelihood $\cdot$ Parental phenotype $\cdot$ Score test $\cdot$ Twin studies

\begin{abstract}
Objective: Standard methods for linkage analysis ignore the phenotype of the parents when they are not genotyped. However, this information can be useful for gene mapping. In this paper we propose methods for age at onset genetic linkage analysis in sibling pairs, taking into account parental age at onset. Methods: Two new score statistics are derived, one from an additive gamma frailty model and one from a log-normal frailty model. The score statistics are classical non-parametric linkage (NPL) statistics weighted by a function of the age at onset of the four family members. The weight depends on information from registries (age-specific incidences) and family studies (sib-sib and father-mother correlation). Results: In order to investigate how age at onset of sibs and their parents affect the information for linkage analysis the weight functions were studied for rare and common disease models, realistic models for breast cancer and human lifespan. We studied the performance of the weighted NPL methods by simulations. As illustration, the score statistics were applied to the GAW12 data. The results show that it is useful to include parental age at onset information in genetic linkage analysis.

Copyright $\odot 2009$ S. Karger AG, Basel
\end{abstract}

\section{Introduction}

A strategy for gene mapping of complex traits is linkage analysis in sibling pairs. For the parents of the siblings information on phenotypes may be available while genotype data are often missing. For example in the Leiden Longevity Study [1], long-lived sibling pairs were genotyped. When the sibling pairs were sampled, the parents were deceased hence ages at death of the parents are known, but no genotypes are available. In addition to the phenotypes of the sib pairs, one also wants to use information on the parental phenotypes. For quantitative traits, information on parental phenotypes can easily be included in the weights of a Haseman-Elston type of analysis [2]. The aim of this paper is to derive these weights for age at onset data. For linkage analysis of age at onset data, multivariate survival models based on frailties have been proposed [3-10]. In these models the alleles at the disease locus are represented by unobserved random effects (frailties) which are transmitted from parents to the children. The indication that different alleles at the disease locus are associated with the disease is measured by the variance of these random effects. In this way, frailty models are variance-component models where the correlation between relatives depends on the number of alleles shared identical-by-descent (IBD) at the putative disease locus. The gamma distribution is most commonly used because of its mathematical tractability [4-9]. In

\section{KARGER}

Fax +4161306 1234

E-Mail karger@karger.ch

www.karger.com
(C) 2009 S. Karger AG, Basel

0001-5652/10/0692-0080\$26.00/0

Accessible online at:

www.karger.com/hhe
Andrea Callegaro

Department of Medical Statistics and Bioinformatics

Leiden University Medical Center, S-5-P, PO Box 9600

NL-2300 RC Leiden (The Netherlands)

Tel. +31 71526 9718, Fax +31 71526 8280, E-Mail a.callegaro@lumc.nl 
particular, Callegaro et al. [9] proposed a robust score test for selected sibling pairs. Their method can be interpreted as a regression of the IBD status of sibling pairs on a function of their ages at onset. The method is applicable to sib pairs selected on the basis of their phenotypes, provided that population parameters (marginal survival and sib-sib correlation) are available. In this paper we extend their method to nuclear families of size 4 (2 parents and an affected sib pair (ASP)).

A drawback of the gamma frailty model is that the corresponding likelihood becomes very complex when the number of relatives increases. In order to solve this problem, we derive also a score test from a log-normal frailty model which can easily be applied to general pedigrees. In the spirit of the work of Lebrec and van Houwelingen [11], the score test is derived from a Taylor approximation of the log-likelihood around random effect equal to zero. The rest of the paper is organized as follows. In the first section, we describe the random effect models. We introduce a general model and describe the structure of the variance-covariance matrix of the random effect. We introduce the gamma and the log-normal frailty models, and the formula of the weights is given. We explore the weights as function of the age at onset of the offspring and of the parents for a rare as well as a common disease. For breast cancer and life span we describe the weights based on available population parameters. To study the performance of the proposed methods we perform a simulation study. Finally, we apply the weighted score tests to the GAW12 simulated data [12].

\section{Methods}

\section{Random Effect Models for Nuclear Families}

Let $T_{i j}$ be the random variable of age at onset for relative $j$ in family $i$. Let $y_{i j}=\left(t_{i j}, \delta_{i j}\right)$ be the observed phenotype data where $t_{i j}$ is the observed age at onset if $\delta_{i j}=1$ and age at censoring if $\delta_{i j}=$ 0 . We assume that all the families consist of two parents $(j=1,2)$ and two children $(j=3,4)$. Let the vector $Y_{i}=\left(t_{i 1}, \delta_{i 1}, t_{i 2}, \delta_{i 2}, t_{i 3}\right.$, $\left.\delta_{i 3}, t_{i 4}, \delta_{i 4}\right)$ be the phenotypes of the 4 pedigree members and let $Z_{i}=\left(Z_{i 1}, Z_{i 2}, Z_{i 3}, Z_{i 4}\right)$ be the vector of random effects (frailties) which models the dependence between the outcomes of relatives. Under the frailty model, the conditional hazard for the $j$-th individual in the $i$-th family given a vector of measured covariates $X_{i}$ and the random effect $Z_{i j}$ is

$$
\lambda\left(t_{i j} \mid X_{i j}, Z_{i j}\right)=\lambda_{0}\left(t_{i j} \mid X_{i j}\right) Z_{i j} \text {, }
$$

where $\lambda_{0}\left(t_{i j} \mid X_{i j}\right)$ is the baseline hazard stratified for the covariates $X_{i j}$. The marginal hazard $h\left(t_{i j} \mid X_{i j}\right)$ is obtained by integrating over the distribution of the random variable $Z_{i j}$. In this paper we assume that the marginal hazard and the frailty parameters $\left(\rho_{s}, \rho_{p}\right.$, $\sigma^{2}$ ) are known from previous studies.

Robust Age at Onset Linkage Analysis in Nuclear Families
For the sake of simpler notation, in the following we drop the family index $i$. Two different distributions of the random effects are used, namely the gamma distribution and the log-normal distribution. For both frailty models, the $(j, k)$-th element of the variance-covariance matrix $(\Sigma=\operatorname{Cov}(Z))$ is given by

$$
\sum(j, k)=\sigma^{2} \begin{cases}a^{2}+c^{2}+e^{2}=1, & \text { if } j=k ; \\ \left(\pi_{j k}-E \pi_{j k}\right) \gamma+\left(E \pi_{j k}\right) a^{2}+c^{2}, & \text { if } j \neq k,\end{cases}
$$

where $\pi_{j k}$ is the proportion of alleles shared IBD between the members $j$ and $k$. The parameter $\gamma$ represents the part of the variance attributable to the linkage effect, $a^{2}$ denotes the part of the variance explained by the additive genetic effects, $c^{2}$ the part of the variance explained by common environmental effects and $e^{2}$ the individual effect. The factor $E \pi_{j k}$ is the expected proportion of alleles shared IBD between pedigree members $j$ and $k$. The advantage of this parametrization of the covariance matrix is that the correlation between two siblings can be written in terms of the marginal correlations $\left(\rho_{s}\right)$ [13]. The correlation between siblings $\left(\rho_{s}(\pi)=(\pi-E \pi) \gamma+\rho_{s}\right)$ depends on the number of alleles shared IBD through the linkage parameter. When the linkage parameter is null $(\gamma=0)$ the correlation is independent of the IBD status, which means that the genetic marker is not linked with a disease locus. Under the null hypothesis of no linkage the sib-sib correlation is the marginal correlation $\left(\rho_{s}\right)$ which is often known from previous twin studies. In contrast with the sib-sib correlation, the sib-parent correlation $\left(\rho_{s p}=\rho_{s}\right)$ and the parent-parent correlations $\left(\rho_{p}=c^{2}\right)$ do not depend on the linkage parameter. This is due to the fact that parent-sibling pairs always share one allele IBD and the two parents do not share any alleles IBD. The likelihood functions $P(Y \mid \pi ; \gamma)$ for the gamma frailty model and for the log-normal frailty model are derived in appendix $A$ and $B$, respectively.

Score Tests

To test the null hypothesis $H_{0}: \gamma=\gamma_{0}=0$ versus $H_{1}: \gamma>0$ a score test is proposed. For a set of $N$ independent pedigrees the score statistic based on the retrospective likelihood of the marker data given the phenotypes (see appendix $\mathrm{C}$ ) is given by

$$
\mathrm{NPL}_{w}=\frac{\sum_{i=1}^{N}\left(\hat{\pi}_{i}-E \hat{\pi}_{i}\right) w_{i}}{\sqrt{\sum_{i=1}^{N} \operatorname{var}_{0}\left(\hat{\pi}_{i}\right) w_{i}^{2}}}
$$

with weight function

$$
w_{i}=\frac{\partial \log P\left(Y_{i} \mid \pi_{i} ; \gamma_{0}\right)}{\partial \rho(\pi)},
$$

where $\log P\left(Y_{i} \mid \pi_{i} ; \gamma\right)$ is the prospective log-likelihood. This score statistic is a weighted nonparametric linkage (NPL) statistic [14] with known weight functions.

The weight derived from the gamma frailty model is a very complex function and the closed form is not reported. These weights depend on marginal frailty parameters $\left(\rho_{s}, \rho_{p}, \sigma_{G}^{2}\right)$ and on the marginal cumulative hazard $H$ (see appendix B for details). The statistic based on this model is denoted by $\mathrm{NPL}_{G}^{p}$. When parental age at onset is not considered $\left(S_{1}=S_{2}=1\right.$ and $\left.\delta_{1}=\delta_{2}=0\right)$ this statistic is equivalent to the statistic derived by Callegaro et al. [9], here denoted by $\mathrm{NPL}_{G}$.

In contrast, the weight derived from the log-normal frailty model assuming small random effects $\left(\mathrm{NPL}_{N}^{p}\right)$ is a simple formu- 
la (see appendices B and C for details). Let $\delta, \Lambda_{0}$ and $M=\left(\delta-\Lambda_{0}\right) /$ $\Lambda_{0}$ be the four-dimensional vectors of the event status, of the baseline cumulative hazards at the age at onset (age at censoring) and of the standardized martingale residuals, respectively. The weight derived from the log-normal frailty model is given by the $(3,4)$-th element of the following matrix

$$
W_{N}=C^{-1} M\left(C^{-1} M\right)^{\prime}-C^{-1},
$$

where $C=D \Sigma D+D$ and $D=\operatorname{diag}\left(\Lambda_{0}\right)$. The weight depends on the frailty parameters $\left(\rho_{s}, \rho_{p}, \sigma_{N}^{2}\right)$ and on the baseline cumulative hazard which is unknown. The estimation of this hazard function is not straightforward when the families have been selected on the phenotypes. In this paper, we estimate the baseline cumulative hazard by the marginal hazard $\left(\hat{\Lambda}_{0}=H\right)$ [15]. This estimator is valid for small residual effects. However, $\mathrm{NPL}_{N}^{p}$ gives actual type I error rates equal to the nominal value, irrespectively of the validity of the baseline estimator.

Note that the classical NPL test [16] corresponds to the score statistic (3) with weight equal to $w_{i}=\delta_{i 3} \times \delta_{i 4}$. In the case of incomplete marker information the variance of the estimated proportion of IBD given the marker data $\left(\operatorname{var}_{0}(\hat{\pi})\right)$ can be estimated by simulations [2].

\section{Results}

\section{Weight Functions}

Weight Function for Common and Rare Disease Models

We used the derived weight functions to describe the relationship between the phenotypes of the nuclear family and the information on linkage. We studied the weight function of the gamma and of the log-normally distributed random effects for a rare and for a common disease. For the rare disease we used a constant marginal hazard rate of 0.001 . The corresponding marginal survival function was equal to 94 and $92 \%$ at age 60 and 80 , respectively. For the common disease a constant marginal hazard rate of 0.01 with marginal survival function equal to 55 and $44 \%$ at age 60 and 80, respectively, was used. The sib-sib and parent-offspring correlations were fixed at 0.5 with a parent-parent correlation of 0.1. Similar results were obtained using different values of these parameters. For the gamma-distributed frailties, we used $\sigma_{G}^{2}$ equal to 1 and to 5. For the log-normally distributed random effects we used $\sigma_{N}^{2}=\log (1+$ $\left.\sigma_{G}^{2}\right)$ to have similar scales for the gamma and normal distributions. For illustration purpose, we used the same age at onset for the siblings. Firstly we considered the situation that both parents had the same age at onset. Secondly we considered the situation that the parents had discordant phenotypes, i.e., one has early onset and the other has late onset of disease.
Figure 1 shows the gamma frailty weight distribution in the case of rare and common disease for $\sigma_{G}^{2}$ equal to 1 and to 5 . When the disease is rare and the variance of the random effect is small parents' age at onsets provides little information (fig. 1a). On the other hand, when the variance increases the weight distribution depends on the age of the parents at onset. Note that the most informative families have discordant siblings-parents phenotypes. In three of the four configurations (fig. 1a, b and c) the most informative families have early-onset siblings and lateonset parents. However, if the variance is not small and the trait is common the most informative families can be the families with late-onset siblings and early-onset parents (fig. 1d).

The weights corresponding to the log-normal distribution are depicted in figure 2 . For small variance, similar results were obtained. For large values of the variance the two approaches gave different results. This is due to the fact that the weights of the normal-distributed frailty are accurate only for small values of the variance. Results may change when parents have different age at onsets. If late-onset siblings have been selected, concordant earlyonset parents are more informative (data not shown). If early-onset siblings have been selected, families with discordant parents appear to be more informative than families with concordant late-onset parents (fig. 3a). If discordant siblings have been selected, the most informative families for linkage are families with early-onset parents (fig. 3b). Note that in this case the weights are always negative because discordant siblings are expected to share less alleles IBD than one.

\section{Weight Function for Breast Cancer}

Wienke et al. [17] modelled the age at onset of breast cancer in Swedish twins data. Using a correlated frailty model with Gompertz-distributed baseline hazard $\left(\lambda_{0}(t)=b e^{s t}\right)$ they estimated the following parameters $\hat{b}=$ $1 / 10^{5}, \hat{\boldsymbol{s}}=0.120, \hat{\sigma}_{G}^{2}=25, \hat{\rho}_{D Z}=0.125$ and $\hat{\rho}_{M Z}=0.154$. From the marginal survival and the two correlation parameters $\left(\rho_{p}=2 \hat{\rho}_{D Z}-\hat{\rho}_{M Z}=0.1\right.$ and $\left.\rho_{s}=\rho_{D Z}=0.125\right)$ we computed the weight function for various age at onset scenarios. The survival of the father was marginalized $(S(t)=1$ and $\delta=0)$ and the two sisters had the same age at onset. Figure $4 \mathrm{a}$ shows the distribution of the gamma frailty weight function in terms of the age at onset of the two sisters and the age at onset of the affected mother. The most informative families have early-onset siblings with late-onset mother. In this case the weight function of the $\mathrm{NPL}_{N}^{p}$ statistic differs from the weight function of the NPL ${ }_{G}^{p}$, because the variance of the random effect is 
Fig. 1. Weights corresponding to the gamma frailty model as a function of the age at onset of the ASP and their affected parents. a Rare disease and $\sigma_{G}^{2}=1$. b Rare disease and $\sigma_{G}^{2}=5$. c Common disease and $\sigma_{G}^{2}=1$. d Common disease and $\sigma_{G}^{2}=5$.
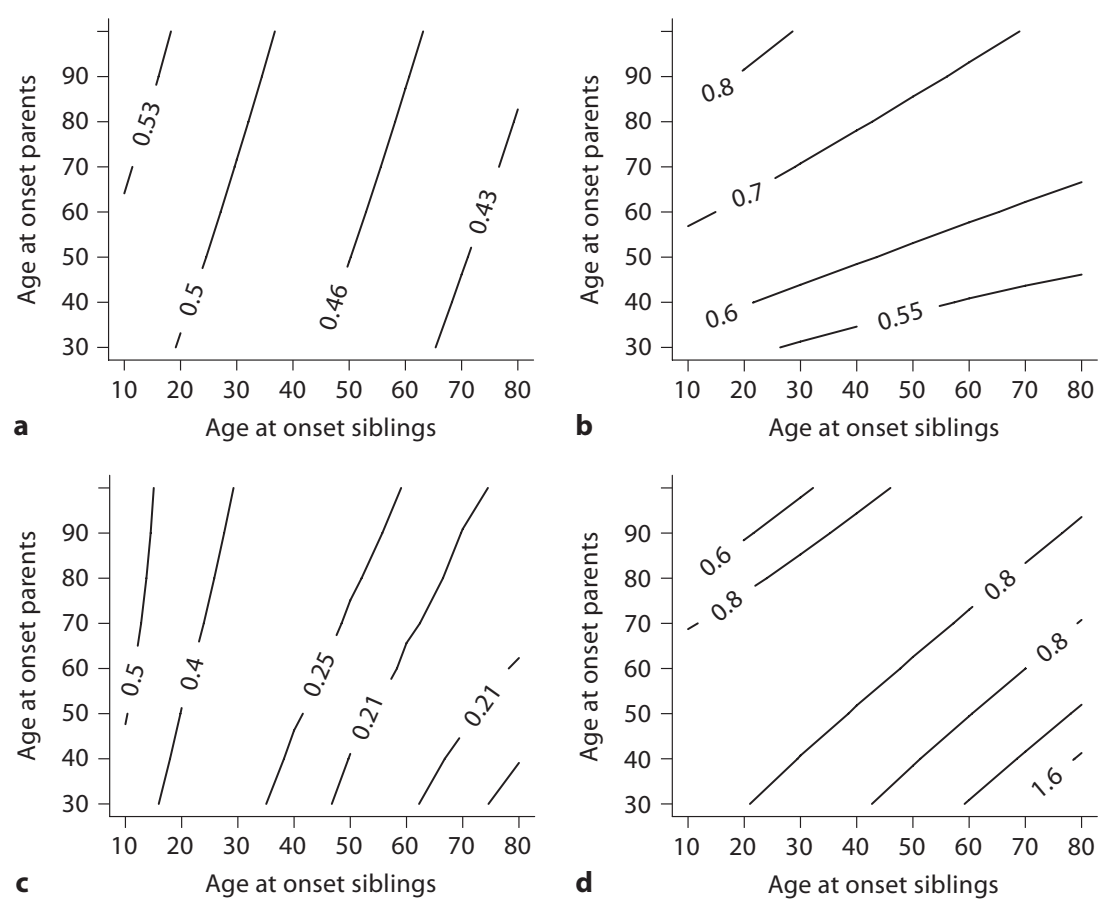

Fig. 2. Weights corresponding to the lognormal model as a function of the age at onset of the ASP and their parents. a Rare disease and $\sigma_{N}^{2}=\log (1+1)$. b Rare disease and $\sigma_{N}^{2}=\log (1+5)$. c Common disease and $\sigma_{N}^{2}=\log (1+1)$. d Common disease and $\sigma_{N}^{2}=\log (1+5)$.

Fig. 3. Weights corresponding to the gamma frailty model as functions of the age at onset of the two parents for a common disease with $\sigma_{G}^{2}=5$. a Early onset ASP. b Discordant sibling pairs.
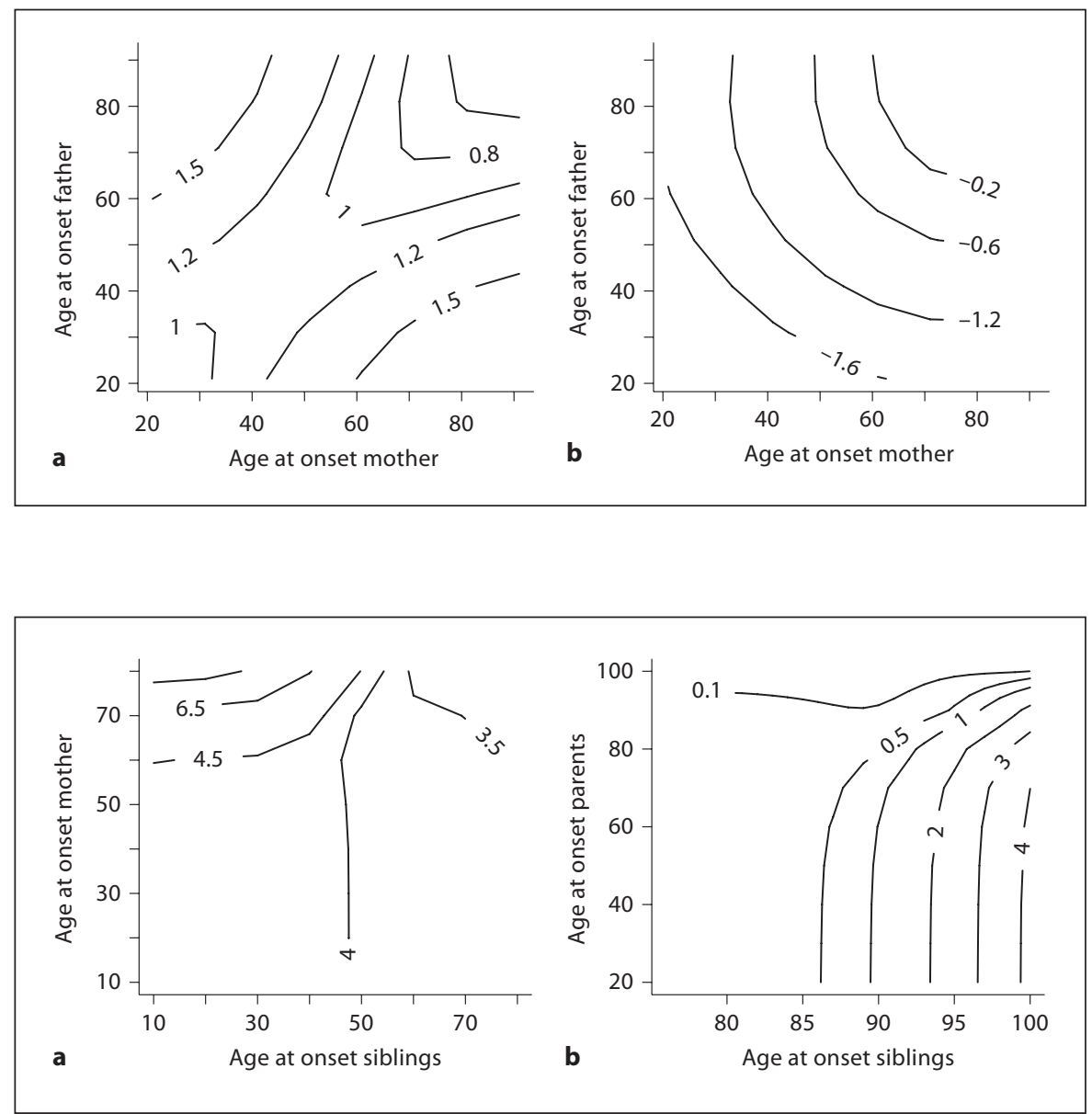

Fig. 4. Weights corresponding to realistic models for breast cancer (a) and for human life span (b). for discordant parents. In fact, the combination of longlived siblings and one early deceased parent is informative for linkage even if the other parent became long-lived (data not shown). The weight function of the $\mathrm{NPL}_{N}^{p}$ is very similar to the weight function of the $\mathrm{NPL}_{G}^{p}$, because the variance is rather small (data not shown).

\section{Simulation Results}

By means of simulations of ASP data we compared the power of the method proposed by Callegaro et al. [9] $\left(\mathrm{NPL}_{G}\right)$ which ignores the parental age at onset to the power of the two new methods proposed in this paper, $\mathrm{NPL}_{G}^{p}$ and $\mathrm{NPL}_{N}^{p}$. Age at onset data were generated based on an additive gamma frailty model with a Gompertzdistributed baseline hazard $\left(\lambda_{0}(t)=b e^{s t}\right)$. Current ages were simulated as age at censoring from a uniform distribution $U(40,100)$. We studied common diseases where almost 10 and $40 \%$ of the population is affected by age 60 and 80 , respectively. We simulated data with two different genetic models with variances of the random effect 
given by $\sigma^{2}=3.3\left(b=1 \times 10^{-5}, \varsigma=0.12\right)$ and $\sigma^{2}=1.1$ $\left(b=1 \times 9^{-5}, s=0.1\right)$, respectively. The random effect was decomposed into the sum of the linkage effect and the residual shared environmental effect. For each of the two genetic models we simulated data according to three decompositions of the total random effect. In the first, the linkage effect explained all the variability of the random effects $(\gamma=1)$. In the second, the linkage effect explained two thirds of the total variance $(\gamma=2 / 3)$. Finally, in the third decomposition of the random effect, the linkage effect explained one third of the total variability $(\gamma=1 / 3)$. Each configuration included 5,000 replications under the null hypothesis and 1,000 replications under the alternative hypothesis. For each replication the first 200 nuclear families with ASPs were ascertained.

We evaluated the performance of the tests with a significance level of $\alpha=0.05$. Under the null hypothesis all the tests have correct type I error (table 1). Under the alternative hypothesis there is an increase in power when incorporating the parental age at onset in the weights (table 2). In the case of large variance of the random effect and small heritability, the gain in power of $\mathrm{NPL}_{G}^{p}$ with respect to $\mathrm{NPL}_{G}$ is about $60 \%$. Note that $\mathrm{NPL}_{G}^{p}$ outperforms $\operatorname{NPL}_{N}^{p}$ in the case of large variance $\sigma^{2}$ because $\mathrm{NPL}_{N}^{p}$ assumes a small variance of the frailty.

We further evaluated the robustness of the proposed methods. Figure 5 shows the power of the proposed score tests in the case of data simulated with $\sigma_{G}^{2}=3.3$ and $\gamma=$ $1 / 3$. The power is shown for different values of the frailty parameters specified in the weight $\left(\sigma^{2}, \rho_{s}, \rho_{p}\right)$. We computed the power as a function of $\sigma^{2}$ for three different values of the heritability $a^{2}=0.25,0.5,0.75$. For simplicity, we defined $c^{2}=1-a^{2} ; \rho_{s}=1 / 2 a^{2}+c^{2}$ and $\rho_{p}=c^{2}$. The horizontal lines represent the unweighted NPL (solid line) which is independent of the frailty parameters. The proposed methods have maximal power around the true population parameter values. However, they outperform the classical NPL methods for a wide range of the frailty parameters used in the weight. For example, NPL ${ }_{G}^{p}$ (fig. 5a) is more powerful than the classical NPL for every value of $\sigma_{G}^{2}$ smaller than 15 . In a similar way, $\mathrm{NPL}_{N}^{p}$ is more powerful than the unweighted method for every value of $\sigma_{N}^{2}$ smaller than 10, irrespectively of the correlation values specified in the weight (fig. $5 b$ ). In this particular case $\mathrm{NPL}_{G}^{p}$ is more powerful than $\mathrm{NPL}_{N}^{p}$ because the data have been simulated with a large variance of the random effect. Note that when the value of the variance specified in the weight is equal to zero $\left(\sigma^{2}=0\right)$ the two proposed methods have the same power, and they are more powerful than the unweighted NPL method.

Robust Age at Onset Linkage Analysis in Nuclear Families
Table 1. Type I error comparisons based on 5,000 replications of ASP with known parental age at onset (significance level $\alpha=0.05$ ). No linkage effect $(\gamma=0)$

\begin{tabular}{lllllllll}
\hline & \multicolumn{3}{l}{$\sigma^{2}=1.1$} & & \multicolumn{2}{l}{$\sigma^{2}=3.3$} \\
\cline { 2 - 4 } \cline { 7 - 9 } & $a^{2}=1$ & $a^{2}=2 / 3$ & $a^{2}=1 / 3$ & & $a^{2}=1$ & $a^{2}=2 / 3$ & $a^{2}=1 / 3$ \\
\hline$N^{N P L}$ & 0.051 & 0.050 & 0.051 & & 0.049 & 0.049 & 0.051 \\
$\mathrm{NPL}_{G}$ & 0.051 & 0.048 & 0.048 & & 0.049 & 0.051 & 0.048 \\
$\mathrm{NPL}_{G}^{p}$ & 0.048 & 0.050 & 0.050 & & 0.049 & 0.049 & 0.049 \\
$\operatorname{NPL}_{N}^{p}$ & 0.051 & 0.047 & 0.051 & & 0.047 & 0.049 & 0.048 \\
\hline
\end{tabular}

Table 2. Power comparison based on 1,000 replications of ASP with known parental age at onset (significance level $\alpha=0.05$ ). No residual additive effect $\left(\gamma=a^{2}\right)$

\begin{tabular}{|c|c|c|c|c|c|c|}
\hline & \multicolumn{3}{|c|}{$\sigma^{2}=1.1$} & \multicolumn{3}{|c|}{$\sigma^{2}=3.3$} \\
\hline & $a^{2}=1$ & $a^{2}=2 / 3$ & $a^{2}=1 / 3$ & $a^{2}=1$ & $a^{2}=2 / 3$ & $a^{2}=1 / 3$ \\
\hline NPL & 0.52 & 0.31 & 0.18 & 0.87 & 0.58 & 0.26 \\
\hline $\mathrm{NPL}_{G}$ & 0.68 & 0.43 & 0.20 & 0.98 & 0.78 & 0.34 \\
\hline $\mathrm{NPL}_{G}^{\tilde{p}}$ & 0.68 & 0.46 & 0.23 & 0.99 & 0.88 & 0.54 \\
\hline $\mathrm{NPL}_{N}^{\vec{p}}$ & 0.67 & 0.44 & 0.21 & 0.98 & 0.79 & 0.36 \\
\hline
\end{tabular}

\section{Application to GAW12 Data}

We applied the proposed methods to the GAW12 simulated data of general population [12]. Disease data were generated using a complex model where seven genes influenced the liability and the age at onset. Major gene 7 directly contributed to age at onset and major gene 6 contributed to the disease liability. Both genes were located on chromosome 6 , at positions 31.5 and $30.5 \mathrm{cM}$, respectively. More details on how the data were generated can be found in [12]. The GAW12 general population data include a total of 50 replicates, each of them containing 23 extended pedigrees. From the first 30 replications we selected 500 independent ASPs with known age at onset (age at censoring) of the parents. The marginal survival functions and the frailty parameters were estimated on the remaining 20 data sets. For the marginal survival, we used a Kaplan-Meier estimator stratified by sex and the frailty parameters by a correlated gamma frailty model $\left(\rho_{s}=0.7, \rho_{p}=0.1, \sigma_{G}^{2}=3.75\right)$. The estimated prevalence of the disease at 70 years is 50 and $25 \%$ for females and males, respectively. We computed the weights for the NPL statistics. For the weights of the NPL $L_{N}^{p}$ we used the variance equal to $\sigma_{N}^{2}=\log \left(1+\sigma_{G}^{2}\right)=1.55$. The weight values of $\mathrm{NPL}_{G}^{p}$ and $\mathrm{NPL}_{N}^{p}$ were quite similar for this data set. As expected, families with early-onset siblings and late- 
Fig. 5. Power of the test statistics based on simulated data (significance level $\alpha=0.05$ ). The horizontal lines represent the power of the unweighted NPL (solid line). Dashed lines, dotted lines, dashed-dotted lines represent the power of proposed NPL methods assuming $a^{2}=0.25,0.5$ and 0.75 , respectively. a $\mathrm{NPL}_{G}^{p}$. b $\mathrm{NPL}_{N}^{p}$.



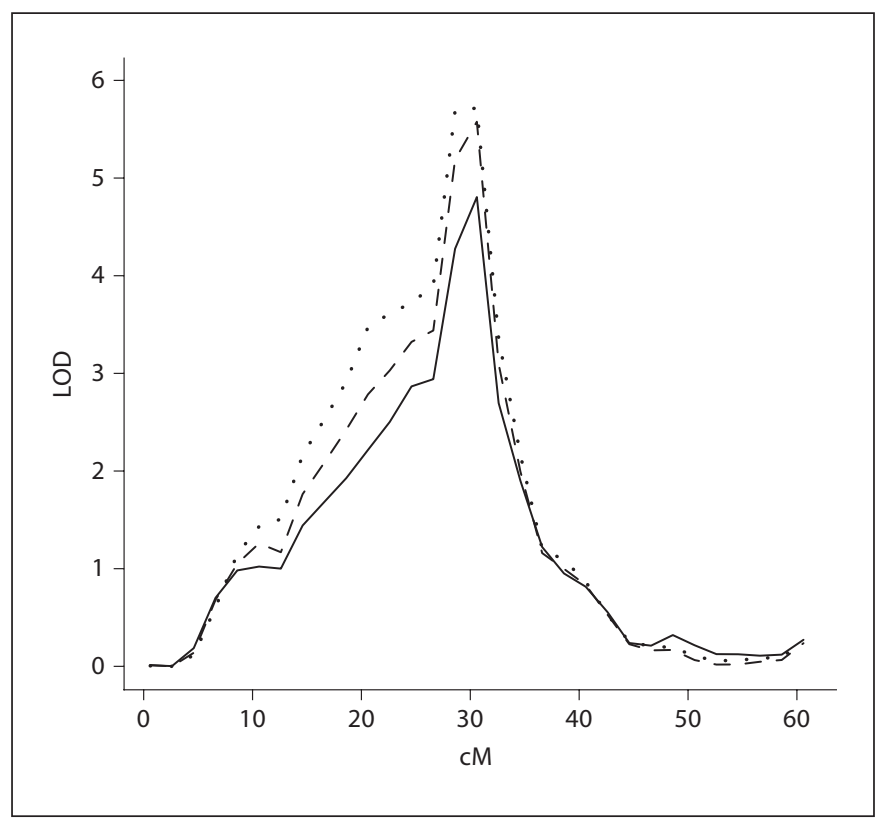

Fig. 6. Genetic linkage analysis of the GAW12 data on chromosome 6. Solid line represents NPL method, dashed line represents $\mathrm{NPL}_{G}^{p}$ method, and dotted line represents $\mathrm{NPL}_{N}^{p}$ method.

onset parents had high values of the weight. However, since we used marginal survival stratified by sex, the values of the weight depend on the sex configuration of the siblings.

Parental genotypes were not considered in the analysis and the variances of the IBD $\left(\operatorname{var}_{0}(\pi)\right)$ were estimated by multipoint simulations using MERLIN $[9,19]$. Without taking into account the age at onset, the most significant evidence for linkage was on chromosome 6, with a NPL LOD score of about 4.5 at the locus of the major gene 7 . Further, we applied the score test of Callegaro et al. [9] $\left(\mathrm{NPL}_{G}\right)$ ignoring the parental ages $(\mathrm{LOD}=5.5)$, and finally we applied the two score tests taking into account the parental age at onset. Figure 6 shows that - at the disease locus $-\mathrm{NPL}_{N}^{p}(\mathrm{LOD}=5.6)$ takes on a slightly larger value than $\mathrm{NPL}_{G}^{p}(\mathrm{LOD}=5.5)$. In this data set incorporating the parental age at onset only slightly changed the results.

\section{Discussion}

In order to map disease genes involved in complex traits one may want to use all available information. A common strategy for linkage is to collect large samples of ASP. In this paper, we extended the score test of Callegaro et al. [9] to include the parental ages at onset or current ages. When frailty parameters are known, the score statistic is a classical NPL statistic [14], with known weights depending on the siblings and on the parental ages at onset. We also derived a score test from a log-normal frailty model. Assuming small random effects, the weight function derived from this model is very simple and it can easily be computed for general sibship sizes.

The weight functions gave us the opportunity to study the complex relationship between age at onset and linkage effect. We explored the weight function for rare and common disease models. In line with previous papers on ASP $[6,9]$ our results show that age at onset is not informative for rare diseases. In the case of common diseases, discordant parent-sibling families appear to be the most 
informative for linkage. A similar result was obtained for quantitative traits [2]. The study of the weight distribution for breast cancer suggests that families with earlyonset sister pairs and late-onset (unaffected) mothers are the most informative families to detect linkage. From the model for human life span our results show that longlived sibling pairs with early deceased parents are the most informative. In fact, selecting ASP with affected parents might increase the number of copies of any given disease-related variant in families. Families carrying multiple copies of a disease-related allele may not show linkage since the affected individuals may inherit that allele from different ancestors and therefore not deviate from expected IBD sharing [20]. Simulation results showed that incorporating the parental age at onset can considerably increase the power to detect linkage. In fact, for a common disease with small heritability, the gain in power of $\mathrm{NPL}_{G}^{p}$ compared to $\mathrm{NPL}_{G}$ was about $60 \%$. We applied the proposed methods to GAW12 simulated data. The NPL scores taking into account the parental age of onset outperformed the NPL method which ignores the age at onset. However, the performance of the proposed methods was similar to the performance of $\mathrm{NPL}_{G}$, which ignores the parental data. This result can be interpreted in terms of the number of families with discordant age at onset in siblings and parents. In fact, only few families were available with early-onset siblings and late-onset parents. Suppose for example that early onset is defined as disease with age of onset before 30 (prevalence of 93\%) and late onset is defined as disease with age of onset/censoring after 60 (prevalence of 50\%). In this case only 10 families out of 500 were available with early-onset siblings and late-onset parents.

The proposed methods can only be applied when population parameters are known. However, the values of these parameters are available for many phenotypes, such as aging [21], breast cancer [17], coronary heart disease [22], malignant melanoma, colon cancer [23], etc. Further, we showed the robustness of these methods against misspecification of the frailty parameters. Simulation results showed that the proposed methods are not very sensitive for the frailty parameter values specified in the weight. The derived score tests have several advantages with respect to likelihood-ratio approaches $[6,7,10,24]$. First of all, they maintain correct type I error irrespectively of the weights. Second, they can easily be applied to selected samples. Finally, they are simpler and computationally faster because they are computed under the null hypothesis.

Robust Age at Onset Linkage Analysis in Nuclear Families
It is interesting to compare the two score tests proposed. The additive gamma frailty model is mathematically appealing because the random effects can be integrated out, which allows us to work with observable marginal survival functions. However, results obtained from these additive models should be interpreted carefully [25]. It may be more intuitive if the variance components act multiplicatively, like unobserved covariates in a Cox model [26]. Another problem is that the likelihood function becomes too complicated to write down for general pedigrees. In contrast, the log-normal model is a multiplicative model. Hence the parameters may be better interpretable. The formula of the weight is simple, and it can easily be computed for sibships of any size. The main limitation of this model is that it is powerful only for small values of the random effects. In this paper, we estimated the baseline hazard with the marginal hazard. A possibility to improve the power of the test based on the log-normal weight is to derive the baseline hazard using numerical methods.

An interesting extension of the proposed models is to include a cure fraction, like in the paper of Wienke et al. [17]. If the cure effect is estimable, these extended models may better explain the data than standard survival methods.

In summary, we derived two different weights for the NPL statistic to take into account the age at onset or current age of two selected siblings and their parents. When the variance of the random effect is large $\left(\sigma_{G}^{2}>3\right)$ we recommend to use the weight based on the gamma frailty model. When the variance of the random effect is small the two proposed methods have similar performance. However, in this case we recommend to use the log-normal weight because the formula is much simpler and it can be computed for sibships of arbitrary sizes.

The relation between the linkage effect, the age at onset of the siblings and their parents is complex and depends on many parameters. This paper is an attempt to understand this relationship and to use it to increase the power to detect linkage. Software to apply the described methods are freely available from our website (http:// www.msbi.nl/genetics).

\section{Acknowledgements}

J.J.H.-D. and A.C. were supported by a grant from the Netherlands Organization for Scientific Research (NWO 917.66.344). GAW12, which made the data available, was supported by grant R01 GM031575.

Hum Hered 2010;69:80-90 


\section{Appendix A}

\section{Additive Gamma Frailty Model}

Let the random effect $Z$ in model (1) be the sum of four independently gamma-distributed random effects $\left(Z=Z_{g}+Z_{p}+U_{c}+\right.$ $\left.U_{e}\right)$. Here $Z_{g, i k} \sim \Gamma\left(\nu_{g}, 1 / \sigma^{2}\right)$ represents the linkage effect, $Z_{p, i k} \sim$ $\Gamma\left(\nu_{p}, 1 / \sigma^{2}\right)$ the residual additive effect, $U_{c, i} \sim \Gamma\left(\nu_{c}, 1 / \sigma^{2}\right)$ the common environment effect and $U_{e, i k} \sim \Gamma\left(\nu_{e}, 1 / \sigma^{2}\right)$ the individual effect. In order to obtain $E(Z)=1$ we assume $\sigma^{2}=1 /\left(\nu_{g}+\nu_{c}+\nu_{p}+\right.$ $\left.\nu_{e}\right)$. Following the work of Commenges [3] the linkage effect is modelled by the sum of two effects which represent the alleles inherited from the parents. Using this model the variance-covariance matrix of the random effects is equal to the classical variance-covariance matrix for quantitative traits (2) where the linkage effect is given by $\gamma=\nu_{g} /\left(\nu_{g}+\nu_{c}+\nu_{p}+\nu_{e}\right)$.

From this model the following four-dimensional marginal survival function can be derived (see below for details):

$$
\begin{aligned}
& S_{1234}(\pi ; \gamma)= \\
& K_{1234}^{-\frac{\rho_{p}}{\sigma^{2}}} \prod_{j=1}^{4} K_{j}^{-\frac{1-2 \rho_{s}+\rho_{p}}{\sigma^{2}}} \prod_{i=1}^{2}\left[K_{i 34} K_{i 3} K_{i 4} K_{i}\right]^{-\frac{1}{2}(1-\tau) \frac{\rho_{s}-\rho_{p}}{\sigma^{2}}} \\
& \times\left[K_{13} K_{14} K_{23} K_{24}\right]^{-\frac{1}{2}} \frac{\rho_{p}-\rho_{s}(\pi)+(1+\tau)\left(\rho_{s}-\rho_{p}\right)}{\sigma^{2}} \\
& \times\left[K_{134} K_{234} K_{1} K_{2}\right]^{-\frac{1}{2}} \frac{\rho_{s}(\pi)-\rho_{p}-(1-\tau)\left(\rho_{s}-\rho_{p}\right)}{\sigma^{2}}
\end{aligned}
$$

where

$$
K_{B}=\sum_{j \in B} \exp \left(\sigma^{2} H_{j}\right)-b+1
$$

with

$$
H_{j}=\int_{0}^{t_{j}} h(u) d u
$$

is the marginal cumulative hazard of the $j$-th subject, $B$ a subset of the indexes $\{1,2,3,4\}$, and the parameter $b$ the number of elements in $B$. The prospective likelihood of the phenotypes $(Y)$ conditional on the proportion of alleles shared IBD $(\pi)$ is a function of (6). In fact, the joint survival and density function for a family with $d=\sum_{j=1}^{4} \delta_{j}$ affected and $4-d$ unaffected relatives is

$$
P(Y \mid \pi ; \gamma)=(-1)^{d} \frac{\partial^{d} S_{1234}(\pi ; \gamma)}{\partial^{\delta_{1}} t_{1} \partial^{\delta_{2}} t_{2} \partial^{\delta_{3}} t_{3} \partial^{\delta_{4}} t_{4}},
$$

where $\partial \delta_{j} t_{j}=\partial t_{j}$ if $\delta_{j}=1$ and $\partial \delta_{j} t_{j}=1$ if $\delta_{j}=0$. The prospective likelihood corresponds to the four-dimensional survival function $S_{1234}(\pi ; \gamma)$ when $d=0$. Note that the frailty parameters $\left(\rho_{s}, \rho_{p}, \sigma^{2}\right)$ can be obtained from twin studies and that under the null hypothesis

$$
\tau=\frac{\nu_{g}}{\nu_{g}+\nu_{p}}=0
$$

and $\rho_{s}(\pi)=\rho_{s}$

Semiparametric Four-Dimensional Survival Function

Arbitrarily label the paternal chromosomes as $(1,2)$ and the maternal chromosomes as $(3,4)$. The inheritance vector of a sib pair is the vector $V_{d}=\left(v_{1}, v_{2}, v_{3}, v_{4}\right)$ where $v_{2 j-1}=1$ or $2, v_{2 j}=3$ or 4 , for $j=1,2$. The inheritance vector indicates which parts of the genome are transmitted to the children from the father and the mother. The linkage effect is modeled by the sum of two allelic effects $\left(U_{g} \sim \Gamma\left(\nu_{g} / 2,1 / \sigma^{2}\right)\right)$. The linkage effect of the father and of the mother are equal to $Z_{g, 1}=U_{g, 1}+U_{g, 2}$ and to $Z_{g, 2}=U_{g, 3}$ $+U_{g, 4}$, respectively. The linkage effect of the $j$-th sibling, $j=3,4$ is equal to $Z_{g, j}=\Sigma_{k=1}^{4} U_{g, k} a_{k j}$, where $a_{k j}=1$ if $\nu_{2(j-2)-1}=k$ or $\nu_{2(j-2)}$ $=k$, and 0 otherwise. The residual additive random effect is modelled as the sum of four random effects $\left(U_{p} \sim \Gamma\left(\nu_{p} / 4,1 / \sigma^{2}\right)\right)$. The residual additive random effect of the father and of the mother are equal to $Z_{p, 1}=U_{p, 1}+U_{p, 2}+U_{p, 3}+U_{p, 4}$ and $Z_{p, 2}=U_{p, 5}+U_{p, 6}+U_{p, 7}$ $+U_{p, 8}$, respectively. The residual additive effects of the two siblings are given by $Z_{p, 3}=U_{p, 1}+U_{p, 2}+U_{p, 5}+U_{p, 6}$ and $Z_{p, 4}=U_{p, 1}+$ $U_{p, 3}+U_{p, 5}+U_{p, 7}$, respectively.

The total random effect of the $j$-th individual, $j=1, \ldots, 4$ is given by the $Z_{j}=Z_{g, j}+Z_{p, j}+Z_{c}+Z_{e, j}$ where $Z_{c} \sim \Gamma\left(\nu_{c}, 1 / \sigma^{2}\right)$ represents the common environmental effect and $Z_{e, j} \sim \Gamma\left(\nu_{c}, 1 / \sigma^{2}\right)$ represents the non-shared environmental effect. by

Then the marginal bivariate survival of two siblings is given

$$
\begin{aligned}
& S_{1234}=E\left[S_{1}^{Z_{1}} S_{2}^{Z_{2}} S_{3}^{Z_{3}} S_{4}^{Z_{4}}\right]= \\
& E\left[\exp \left(-U_{c} \sum_{j=1}^{4} \Lambda_{j}\right) \prod_{j=1}^{4} \exp \left(-U_{e, j} \Lambda_{j}\right)\right. \\
& \exp \left(-U_{p, 1}\left(\Lambda_{1}+\Lambda_{3}+\Lambda_{4}\right)\right) \exp \left(-U_{p, 2}\left(\Lambda_{2}+\Lambda_{3}+\Lambda_{4}\right)\right) \\
& \exp \left(-U_{p, 3}\left(\Lambda_{1}+\Lambda_{3}\right)\right) \exp \left(-U_{p, 4}\left(\Lambda_{2}+\Lambda_{3}\right)\right) \\
& \exp \left(-U_{p, 5}\left(\Lambda_{1}+\Lambda_{4}\right)\right) \exp \left(-U_{p, 6}\left(\Lambda_{2}+\Lambda_{4}\right)\right) \\
& \exp \left(-U_{p, 7} \Lambda_{1}\right) \exp \left(-U_{p, 8} \Lambda_{2}\right) \exp \left(-U_{g, 1}\left(\Lambda_{1}+a_{13} \Lambda_{3}+a_{14} \Lambda_{4}\right)\right) \\
& \exp \left(-U_{g, 2}\left(\Lambda_{1}+a_{23} \Lambda_{3}+a_{24} \Lambda_{4}\right)\right) \exp \left(-U_{g, 3}\left(\Lambda_{2}+a_{33} \Lambda_{3}+a_{34} \Lambda_{4}\right)\right. \\
& \left.\exp \left(-U_{g, 4}\left(\Lambda_{2}+a_{43} \Lambda_{3}+a_{44} \Lambda_{4}\right)\right)\right] . \\
& S_{1234}=\left(1+\sigma^{2} \sum_{j=1}^{4} \Lambda_{j}\right)^{-\nu_{c}}\left[\prod_{j=1}^{4}\left(1+\sigma^{2} \Lambda_{j}\right)\right]^{-\nu_{e}} \\
& {\left[\prod_{i=1}^{2}\left(1+\sigma^{2}\left(\Lambda_{i}+\Lambda_{3}+\Lambda_{4}\right)\right)\left(1+\sigma^{2}\left(\Lambda_{i}+\Lambda_{3}\right)\right)\right.} \\
& \left.\left(1+\sigma^{2}\left(\Lambda_{i}+\Lambda_{4}\right)\right)\left(1+\sigma^{2}\left(\Lambda_{i}\right)\right)\right]^{-\frac{\nu_{p}}{4}} \\
& {\left[\left(1+\sigma^{2}\left(\Lambda_{1}+a_{13} \Lambda_{3}+a_{14} \Lambda_{4}\right)\right)\left(1+\sigma^{2}\left(\Lambda_{1}+a_{23} \Lambda_{3}+a_{24} \Lambda_{4}\right)\right)\right.} \\
& \left.\left(1+\sigma^{2}\left(\Lambda_{2}+a_{33} \Lambda_{3}+a_{34} \Lambda_{4}\right)\right)\left(1+\sigma^{2}\left(\Lambda_{2}+a_{43} \Lambda_{3}+a_{44} \Lambda_{4}\right)\right)\right]^{-\frac{\nu_{g}}{2}}
\end{aligned}
$$

In the following we apply the transformation $\Lambda_{j}=\left(S_{j}^{-\sigma^{2}}-1\right) / \sigma^{2}$ where $S_{j}$ is the marginal survival of the $j$-th subject. If we define $K_{B}=\Sigma_{j \in B} \exp \left(\sigma^{2} H_{j}\right)-b+1, B$ is a subset of the elements $(1,2,3$, 4 ) and $b$ is the number of elements in $B$ then the survival functions for 0 and 1 in proportion of alleles shared IBD are given by

$$
\begin{aligned}
& S_{1234 \mid \pi=0}=K_{1234}^{-\nu_{c}} \prod_{j=1}^{4} K_{j}^{-\nu_{e}} \prod_{i=1}^{2}\left[K_{i 34} K_{i 3} K_{i 4} K_{i}\right]^{-\nu_{p} / 4}\left[K_{13} K_{14} K_{23} K_{24}\right]^{-\nu_{g} / 2} . \\
& S_{1234 \mid \pi=1}=K_{1234}^{-\nu_{c}} \prod_{j=1}^{4} K_{j}^{-\nu_{e}} \prod_{i=1}^{2}\left[K_{i 34} K_{i 3} K_{i 4} K_{i}\right]^{-\nu_{p} / 4}\left[K_{134} K_{234} K_{1} K_{2}\right]^{-\nu_{g} / 2} .
\end{aligned}
$$


If the parental genotypes are unknown then the survival conditioned on half of the alleles shared IBD is the mean of the two survival functions

$$
\begin{aligned}
S_{1234 \mid \pi=0.5}= & K_{1234}^{-\nu_{c}} \prod_{j=1}^{4} K_{j}^{-\nu_{e}} \prod_{i=1}^{2}\left[K_{i 34} K_{i 3} K_{i 4} K_{i}\right]^{-\nu_{p} / 4} \times \\
& \frac{\left[K_{134} K_{23} K_{24} K_{1}\right]^{-\nu_{g} / 4}+\left[K_{234} K_{13} K_{14} K_{2}\right]^{-\nu_{g} / 2}}{2} .
\end{aligned}
$$

In order to simplify the formula, we approximate the arithmetic mean with a geometric mean

$$
S_{1234 \mid \pi=0.5} \approx K_{1234}^{-\nu_{c}} \prod_{j=1}^{4} K_{j}^{-\nu_{e}} \prod_{i=1}^{2}\left[K_{i 34} K_{i 3} K_{i 4} K_{i}\right]^{-\nu_{p} / 4-\nu_{g} / 4} .
$$
by

If follows that the approximated $4 \mathrm{D}$ survival function is given

$$
\begin{aligned}
S_{1234}= & K_{1234}^{-\nu_{c}} \prod_{j=1}^{4} K_{j}^{-\nu_{e}} \prod_{i=1}^{2}\left[K_{i 34} K_{i 3} K_{i 4} K_{i}\right]^{-\frac{\nu_{p}}{4}} \times \\
& {\left[K_{13} K_{14} K_{23} K_{24}\right]^{-\frac{\nu_{g}(1-\pi)}{2}}\left[K_{134} K_{234} K_{1} K_{2}\right]^{-\frac{\nu_{g} \pi}{2}} . }
\end{aligned}
$$

\section{Appendix B}

\section{Log-Normal Frailty Model}

Let $\delta, \Lambda_{0}$ and $V=\log Z$ be the four-dimensional vectors of the status, of the baseline cumulative hazards at the age at onset (age at censoring) and the normally distributed random effects of the four pedigree members, respectively. The random effect $V$ follows a multivariate normal distribution with mean zero and covariance matrix $\Sigma$ (2). The log-likelihood can be approximated by using a second order Taylor approximation around $V=0$. For small $\sigma_{V}^{2}$, it follows that

$$
\log P\left(Y \mid \Lambda_{0}, V\right) \approx \sum_{i=1}^{4} c_{i}-\frac{1}{2} \Lambda_{0, i}\left(\frac{\delta_{i}-\Lambda_{0 i}}{\Lambda_{0 i}}-V_{i}\right)^{2} .
$$

When the baseline cumulative hazard is known, the vector of standardized phenotypes behaves as a normal distribution [15]. Integrating over the distribution of the random effect gives

$$
\frac{\delta-\Lambda_{0}}{\Lambda_{0}} \sim N\left(0, \sum+\operatorname{diag}\left(1 / \Lambda_{0}\right)\right),
$$

and the vector of the baseline martingale residuals is distributed as

$$
M=\delta-\Lambda_{0} \sim N(0, D \Sigma D+D),
$$

where $D=\operatorname{diag}\left(\Lambda_{0}\right)$. It follows that for small $\sigma_{V}^{2}$, the four-dimensional likelihood is given by

$$
P(Y \mid \pi ; \gamma) \approx|C|^{-1 / 2} \exp \left(-\frac{1}{2} M^{\prime} C^{-1} M\right),
$$

where $C=D \Sigma D+D$.

\section{Appendix C}

Retrospective Likelihood

Using Bayes' rule the retrospective log-likelihood of the marker data (MD) given the phenotype $Y[6,27]$ is given by

$$
\begin{aligned}
\ell(\gamma) & =\log P(\operatorname{MD} \mid Y ; \gamma) \\
& =\log \left[\sum_{\pi \in\{0,0.5,1\}} P(Y \mid \pi ; \gamma) g_{\pi}\right] \\
& -\log \left[\sum_{\pi \in\{0,0.5,1\}} P(Y \mid \pi ; \gamma) p_{\pi}\right]+\log P_{0}(\mathrm{MD}),
\end{aligned}
$$

where $g \pi=P_{0}(\pi \mid \mathrm{MD}), p \pi=P_{0}(\pi)$, and $P_{0}$ is the probability assuming independent assortment of chromosomal regions to gametes.

\section{References}

1 Beekman M, Blauw GJ, Houwing-Duistermaat JJ, Brandt BW, Westendorp RGJ, Slagboom PE: Chromosome 4q25, microsomal transfer protein gene, and human longevity: novel data and a meta-analysis of association studies. J Gerontol A Biol Sci Med Sci 2006; 61:355-362.

2 Lebrec J, Putter H, Houwelingen JC: Score test for detecting linkage to complex traits in selected samples. Genet Epidemiol 2004;27: 97-108.

3 Commenges D: Robust genetic linkage analysis based on a score test of homogeneity: the weighted pairwise correlation statistic. Genet Epidemiol 1994;11:189-200.

$4 \mathrm{Li} \mathrm{H}$ : The additive genetic gamma frailty model for linkage analysis of age-of-onset variation. Ann Hum Genet 1999;63:455468.
Siegmund KD, Todorov AA: Linkage analysis for diseases with variable age of onset. Hum Hered 2000;50:205-210.

-6 Li H, Zhong X: Multivariate survival models induced by genetic frailties, with application to linkage analysis. Biostatistics 2002;3:5775.

$7 \mathrm{Li} \mathrm{H}$ : An additive genetic gamma frailty model for linkage analysis of diseases with variable age of onset using nuclear families. Lifetime Data Anal 2002;8:315-334.

8 Houwing-Duistermaat JJ, Callegaro A, Beekman M, Westendorp RG, Slagboom PE, van Houwelingen JC: Weighted statistics for aggregation and linkage analysis of human longevity in selected families: The Leiden Longevity Study. Stat Med 2008;28:140-151.
Callegaro A, van Houwelingen HC, Houwing-Duistermaat JJ: Score test for age at onset genetic linkage analysis in selected sibling-pairs. Stat Med 2009;28:1913-1926.

10 Pankratz VS, de Andrade M, Therneau TM: Random-effects Cox proportional hazards model: general variance components methods for time-to-event data. Genet Epidemiol 2005;28:97-109.

11 Lebrec JJ, van Houwelingen HC: Score test for linkage in generalized linear models. Hum Hered 2007;64:5-15.

12 Almasy L, Terwilliger JD, Nielsen D, Dyer TD, Zaykin D, Blangero J: GAW12: Simulated genome scan, sequence, and family data for a common disease. Genet Epidemiol 2001;21S:332-338. 
-13 Tang HK, Siegmund D: Mapping quantitative trait loci in oligogenic models. Biostatistics 2001;2:147-162.

14 Kruglyak L, Daly MJ, Reeve-Daly MP, Lander ES: Parametric and nonparametric linkage analysis: a unified multipoint approach. Am J Hum Genet 1996;58:1347-1363.

15 Wintrebert CM, Zwinderman AH, Maat-Kievit A, Roos RA, van Houwelingen HC: Assessing genetic effects in survival data by correlating martingale residuals with an application to age at onset of Huntington disease. Stat Med 2006;25:3190-3200.

-16 Blackwelder WC, Elston RC: A comparison of sib-pair linkage tests for disease susceptibility loci. Genet Epidemiol 1985;2:85-97.

17 Wienke A, Lichtenstein P, Yashin AI: A bivariate frailty model with a cure fraction for modeling familial correlations in diseases. Biometrics 2003;59:1178-1183.
18 Yashin AI, Begun AZ, Iachine IA: Genetic factors in susceptibility to death: a comparative analysis of bivariate survival models. J Epidemiol Biostat 1999;4:53-60.

19 Abecasis GR, Cherny SS, Cookson WO, Cardon LR: Merlin-rapid analysis of dense genetic maps using sparse gene flow trees. Nat Genet 2002;30:97-101.

20 Wallace C, Clayton DG: Appropriate use of information on family history of disease in recruitment for linkage analysis studies. Ann Hum Genet 2006;70:360-371.

-21 Yashin AI, Vaupel JW, Iachine IA: Correlated individual frailty: an advantageous approach to survival analysis of bivariate data. Math Popul Stud1995;5:145-159.
22 Zdravkovic S, Wienke A, Pedersen NL, Marenberg ME, Yashin AI, De Faire U: Heritability of death from coronary heart disease: a 36-year follow-up of 20,966 Swedish twins. J Intern Med 2002;252:247-254.

23 Zahl PH: Frailty modelling for the excess hazard. Stat Med 1997;16:1573-1785.

24 Jonker MA, Bhulai S, Boomsma DI, Ligthart RS, Posthuma D, Van der Vaart AW: Gamma frailty model for linkage analysis with application to interval-censored migraine data. Biostatistics 2008;10:187-200.

25 Petersen JH, Andersen PK, Gill RD: Variance components models for survival data. Stat Neerl 1996;50:193-211.

-26 Cox DR: Regression models and life tables. J R Stat Soc Series B Stat Methodol 1972;34: 187-220.

$>27$ Whittemore AS: Genome scanning for linkage: an overview. Am J Hum Genet 1996;59: 704-716. 